\title{
Length of stay in ICU of Covid-19 patients in England, March - May 2020
}

DOI:

https://doi.org/10.23889/ijpds.v5i4.1411

10.23889/IJPDS.V5|4.1411

10.23889/ijpds.v5i4.1411

Document Version

Final published version

Link to publication record in Manchester Research Explorer

\section{Citation for published version (APA):}

Shryane, N., Pampaka, M., Aparicio Castro, A., Ahmad, S., Elliot, M., Kim, J., Murphy, J., Olsen, W., Perez Ruiz, D., \& Winiowski, A. (2021). Length of stay in ICU of Covid-19 patients in England, March - May 2020. International Journal of Population Data Science, 5(4), 1411. [05]. https://doi.org/10.23889/ijpds.v5i4.1411, https://doi.org/10.23889/IJPDS.V5I4.1411, https://doi.org/10.23889/ijpds.v5i4.1411

\section{Published in:}

International Journal of Population Data Science

\section{Citing this paper}

Please note that where the full-text provided on Manchester Research Explorer is the Author Accepted Manuscript or Proof version this may differ from the final Published version. If citing, it is advised that you check and use the publisher's definitive version.

\section{General rights}

Copyright and moral rights for the publications made accessible in the Research Explorer are retained by the authors and/or other copyright owners and it is a condition of accessing publications that users recognise and abide by the legal requirements associated with these rights.

\section{Takedown policy}

If you believe that this document breaches copyright please refer to the University of Manchester's Takedown Procedures [http://man.ac.uk/04Y6Bo] or contact uml.scholarlycommunications@manchester.ac.uk providing relevant details, so we can investigate your claim.

\section{OPEN ACCESS}




\section{International Journal of Population Data Science}

\section{Length of Stay in ICU of Covid-19 patients in England, March - May 2020}

Nick Shryane ${ }^{1}$, Maria Pampaka ${ }^{1}$, Andrea Aparicio-Castro ${ }^{1}$, Shazaad Ahmad ${ }^{2}$, Mark J Elliot ${ }^{1}$, Jihye Kim ${ }^{1}$, Jennifer Murphy ${ }^{1}$, Wendy Olsen ${ }^{1}$, Diego Pérez Ruiz ${ }^{1}$, and Arkadiusz Wiśniowski ${ }^{1,{ }^{*}}$

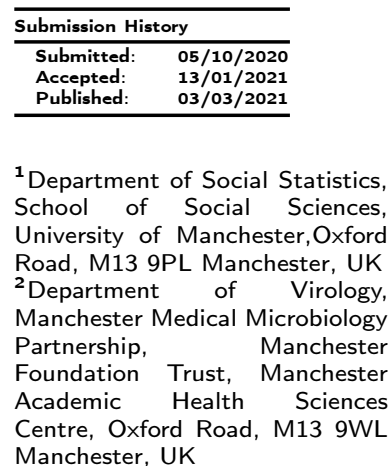

Manchester, UK

\begin{abstract}
Introduction

Length of Stay (LoS) in Intensive Care Units (ICUs) is an important measure for planning beds capacity during the Covid-19 pandemic. However, as the pandemic progresses and we learn more about the disease, treatment and subsequent LoS in ICU may change.
\end{abstract}

\section{Objectives}

To investigate the LoS in ICUs in England associated with Covid-19, correcting for censoring, and to evaluate the effect of known predictors of Covid-19 outcomes on ICU LoS.

\section{Data sources}

We used retrospective data on Covid-19 patients, admitted to ICU between 6 March and 24 May, from the "Covid-19 Hospitalisation in England Surveillance System" (CHESS) database, collected daily from England's National Health Service, and collated by Public Health England.

\section{Methods}

We used Accelerated Failure Time survival models with Weibull and log-normal distributional assumptions to investigate the effect of predictors, which are known to be associated with poor Covid-19 outcomes, on the LoS in ICU.

\section{Results}

Patients admitted before 25 March had significantly longer LoS in ICU (mean $=18.4$ days, median =12), controlling for age, sex, whether the patient received Extracorporeal Membrane Oxygenation, and a co-morbid risk factors score, compared with the period after 7 April (mean $=15.4$, median $=10$ ). The periods of admission reflected the changes in the ICU admission policy in England. Patients aged 50-65 had the longest LoS, while higher co-morbid risk factors score led to shorter LoS. Sex and ethnicity were not associated with ICU LoS.

\section{Conclusions}

The skew of the predicted LoS suggests that a mean LoS, as compared with median, might be better suited as a measure used to assess and plan ICU beds capacity. This is important for the ongoing second and any future waves of Covid-19 cases and potential pressure on the ICU resources. Also, changes in the ICU admission policy are likely to be confounded with improvements in clinical knowledge of Covid-19.

\section{Keywords}

COVID-19; length of stay; intensive care; survival analysis; England

\footnotetext{
*Corresponding Author:

Email Address: a.wisniowski@manchester.ac.uk (Arkadiusz Wiśniowski)
} 


\section{Introduction}

\section{Background}

As of 1 February 2021, more than 103 million people worldwide had tested positive for SARS-CoV-2, with over 2.2 million deaths due to Covid-19 $1{ }^{1}$ 93,448 deaths within 28 days from a positive test were reported in England ${ }^{2}$. Numerous efforts have been undertaken by epidemiologists to study the spread of the pandemic [1, 2], fatality rates [3] and the effectiveness of non-pharmaceutical interventions such as mitigation and suppression [4]. Several medical and public health studies have highlighted the increased length of stay (LoS) in Intensive Care Units (ICUs) [5-8].

Some infected people are asymptomatic [9], others have symptoms of varying severity, with a significant minority requiring ICU admission (5\% of all of those testing positive in China [10]; $12 \%$ in Italy [5]). Such rates can lead to a fast depletion of ICU capacity, especially with increasing prevalence of Covid-19 in the population (idem). This information is relevant during the decision-making process on admission to ICUs, and also for the management and planning of resource allocation in the health care system [11]. Hence, it is important to know the average of and expected variation in ICU LoS, and to determine which factors might predict that variation.

Early studies of ICU LoS for Covid-19 restricted themselves to reporting descriptive statistics. A median of nine days was reported in an Italian study of 1,591 ICU patients with an average age of sixty-three [5]. In China, a median of eight days in ICU was found based on a compilation of 46 different studies [12]. The overall median conceals a wide variation in LoS, from a median of five days for patients who had died in ICU $[13,14]$ to a LoS of twenty-eight days for patients aged over sixty who had not died during the study [14]. For total hospital stay (including ICU), a median of twelve days (mean 12.8) was found for 1,099 Covid-19 patients in China with an average age of fifty-two [15]. In England, a median of twelve days for survivors and ninety days for non-survivors were reported as of 3 July 2020 [7].

For all the aforementioned studies, the complete LoS was calculated only for patients who had already died or been discharged from ICU; the LoS estimates were not adjusted for censoring, i.e. for the fact that patients still in ICU may go on to have greater LoS than that observed during the data collection window of the study. For example, $94 \%$ of those reported by [15] were still in hospital and were not included in calculating LoS. ICU LoS reported by [5] included $58 \%$ of the patients still in ICU but did not correct for censoring. LoS reported by Intensive Care National Audit \& Research Centre (ICNARC) [7] was based on 9,768 patients with 519 still receiving critical care. Therefore, these figures on ICU LoS for Covid-19 are likely to be underestimates.

\section{Aims}

Our aim was to provide more accurate estimates of the ICU LoS attributable to Covid-19. We analysed the LoS in

$$
\begin{aligned}
& \text { ICU } \\
& \hline \text { 1 ht } \\
& { }^{2} \mathrm{ht} \\
& { }^{3} \mathrm{ht} \\
& \mathrm{m} \\
& { }^{4} \mathrm{~T} \\
& 2
\end{aligned}
$$


Shryane, N et. al. / International Journal of Population Data Science (2021) 5:4:05

Table 1: Number of records of ICU stays by final outcome and when the outcome happened

\begin{tabular}{lcccr}
\hline Final Outcome & While in ICU & After ICU stay & Unknown & Total \\
\hline Death & 1,350 & 83 & 1 & 1,434 \\
Discharged & 843 & 1,020 & 1 & 1,864 \\
Censored & 222 & 137 & 384 & 743 \\
Total & 2,415 & 1,240 & 386 & 4,041 \\
\hline
\end{tabular}

Source: own elaboration using CHESS data until 26 May 2020. Note: the final outcome denotes the outcome that could have happened after a patient left ICU.

still in ICU at the end of the data collection period, or had an incomplete record which omitted the final outcome. For these latter cases, we assumed that the last date on which the patient's CHESS record had been updated, or if this was not available the date of the most recent SARS-Cov2 test swab (after they had been admitted to ICU), was the last date they were definitely still in ICU $(n=222)$ as this date is the last time we actually observe the patient to be in ICU. Thus, we treated these patients as having censored ICU LoS. Those patients might, in fact, have left the ICU but the data do not contain information on such an event.

Records for which LoS could not be calculated $(n=386)$, or for which negative $\operatorname{LoS}(n=13)$ or zero LoS $(n=48)$ were implied by the data were removed from the analysis, leaving a sample of 3,594 cases. After also removing cases with missing predictor information on health risk factors score $(n=545)$, a final sample of $n=3,049$ was used. Table B1 presents the LoS a recorded in the final sample broken by characteristics described in further sections.

\section{Patient-level pre-morbid predictor variables}

We found no studies specifically focused on evaluating risk factors for ICU LoS due to Covid-19, although associations have been found for non Covid-19 ICU LoS (see for example [20]) and Covid-19-related hospital (not exclusively ICU) LoS [21]. Instead, we looked at studies that evaluated pre-morbid, individual level risk factors for Covid-19 severity and mortality. Of these, the most consistently reported were old age, male sex, and the presence of comorbid conditions [22-25]. We used these as the primary predictors of LoS in our analyses. In our sample, there were 920 (30.2\%) females and 2,129 (69.8\%) males. We grouped age into four categories: $18-49$ years old $(n=682), 50-64$ years old $(n=$ $1,322), 65-74$ years old $(n=726), 75$ or over $(n=319)$. We further evaluated whether there might have been differential effects of age on LoS by sex, as well as the effects of age and sex independently.

We also explored differences in LoS by ethnicity. These analyses were exploratory because of the amount of missing ethnicity data $(n=535)$ and the consequent potential for biased findings. Ethnicity was grouped into five categories (based on those used by the UK Office for National Statistics): Black (including black African and Afro-Caribbean; $n=134$ ), Asian (primarily Indian, Pakistani, and Bangladeshi; $n=388$ ), Mixed $(n=51)$, Other $(n=162)$, and White $(n=1,779)$. The White group was used as a baseline category in the analysis.

\section{Risk factor score}

We used information on eleven individual health-related risk factors recorded in CHESS: asthma (requiring medication), diabetes (I and II combined), chronic heart disease, hypertension, immunosuppression due to disease, immunosuppression due to treatment, chronic liver disease, chronic neurological disease, chronic renal disease, respiratory disease, and clinician-rated obesity; all were coded as binary items ( $0=$ no, $1=$ yes $)$, apart from clinician-rated obesity which was coded on a three-point scale: $(0=$ no, $1=$ maybe, $2=$ yes $)$. The data on risk factors was incomplete; specifically, there was often no distinction between the patient not having a condition and the information being missing due to not being recorded. To make maximum use of the available information, we used the one-parameter Rasch partial credit model to combine the information from all of the risk factors into one overall score. This model allowed us to test whether the risk factors formed a uni-dimensional scale and to account for their intercorrelations (e.g. heart disease and hypertension were highly correlated) [26]. The Rasch risk score summarised the pre-existing health-statuses and comorbid conditions of the patient, a high score indicating the presence of many health-related diseases and risk factors, and a low score indicating lower risks. Details of the Rasch model results are in Appendix A.3.

\section{Treatment-related factors}

Some studies have reported on post ICU-admission factors (e.g. mode of respiratory support, fraction of inspired oxygen, secondary infections) influencing outcome severity [5]. The secondary infections indicate that they might be acquired in general ward or intensive care (Hospital-Acquired Infection; $\mathrm{HAI}$ ). Typically, these infections lead to longer LoS [27]. There were 580 cases with secondary bacterial pneumonia or other infections in our sample, and they had, on average, longer LoS in ICU than other patients (19.3 vs. 11.6 days for patients with an outcome recorded). However, only 125 cases had a swab for that infection after they were admitted to the ICU, which we believe suggests it was acquired in ICU. This was assessed by comparing the swab date for secondary infection with the dates of admission and leaving of the ICU. We decided not to use the treatment-related factors as predictors because they will not be available before the patient is admitted to ICU, and they likely reflect a complex interaction between unobserved patient characteristics, the severity of infection and the treatment choices made by the clinicians, which would render these predictors endogenous to the outcome. 
The single exception to this decision was the inclusion of whether the patient received Extracorporeal Membrane Oxygenation (ECMO) while in ICU. Previous studies have shown that ECMO patients had longer LOS in ICU because of the severity of their condition and the nature of the treatment $[28,29]$. To complicate this further, ECMO was only available in a few hospitals in England. For these reasons, we included an indicator variable for whether a patient received ECMO $(n=73)$ in our analyses.

\section{Accounting for period effects: admission period, ICU entry guidelines and guidance on proning}

We used data from a two-month period when Covid-19 infections were rising rapidly to a peak in England, approaching and nearly overwhelming critical-care capacity in some areas. During this time there was a change in official health-service guidelines on the criteria to be used in assessing Covid-19 patients for suitability of ICU admission $[16]^{5}$. The nature of the change in guidelines was to give more weight to frailty rather than age of the patient when considering whether they might benefit from treatment in ICU. As the pandemic progressed, clinicians gained experience in treating Covid-19 patients, reflected in the guidance on proning of conscious patients on 12 April 2020 [17] to improve their oxygenation and hence survivorship [30]. All of these factors are likely to have had an impact on the characteristics of patients being admitted to ICU and decisions on who and when to discharge from ICU.

To account for these period-effects on LoS we divided the data collection window into three periods, focusing on the change of the ICU entry guidelines: i) pre-change, i.e. until 24 March 2020, for ICU admissions before the guideline update $(N=387)$, ii) transition, covering ICU admissions from 25 March to 7 April $2020(n=1,452)$ during the transition in guidelines, and iii) post-change, for ICU admissions from 8 April to 24 May $2020(n=1,210)$.

We hypothesised that the changes over time might have had a differential effect on patients based upon their age and frailty. We did not have a direct measure of frailty, so we used the health-related risk factor score as a proxy variable, which is likely to be positively correlated with frailty (i.e. a patient with many risk factors is unlikely to have a low frailty score).

\section{Statistical analysis}

We used parametric, continuous-time survival models, also known as Accelerated Failure Time (AFT) models, to evaluate the LoS from ICU admission until the patient left ICU (by death, discharge, or transfer). We used the AFT models to evaluate how the above-described predictor variables lengthen or shorten LoS. These models can also allow for patients who were still in ICU and who had therefore not yet reached their full LoS (i.e. censored observations). This is achieved by making an assumption about the distributional form of the underlying hazard. The hazard is the probability that leaving ICU will occur at a particular time, given that it has not already occurred. In our models, we evaluated two commonly used parametric hazard distributions: the Weibull and the log-normal. The Weibull distribution can allow for hazards that are either stable, or monotonically increasing or decreasing over time. The log-normal can allow for hazards that are nonmonotonic, i.e. the risk of observing an event for a patient first increases until a peak, then decays. We fitted these AFT models by maximum likelihood estimation. We used the Stata 16 command streg [31]. Code for the method is available at https://github.com/a-wis/CHESS-covid19-los.

We divided our analyses into two sets. In Set 1 we evaluated models of LoS that did not include ethnicity as a predictor. In this set we compared models of the main effects of the predictor variables above, with models that also included the three interaction effects: (admission period)*age, (admission period)*(risk factor score), and sex*age. We also evaluated the choice of baseline hazard function, i.e. Weibull or log-normal. The goodness-of-fit of these models were compared using Akaike's Information Criterion (AIC), which is a penalised likelihood suitable for comparing complex multivariate models because it adjusts for model complexity and the likely fit of the model in alternative samples of data [32]

In Set 2 we evaluated the effect of including ethnicity as a predictor variable into the preferred model from Set 1 . The large amount of missing data for the ethnicity variable meant that the sample size was smaller for Set 2 models compared to Set 1 models, which might lead to biased estimates of LoS. To evaluate if this smaller sample biased our LoS estimates from Set 1, we also fitted the preferred Set 1 model (without ethnicity as a predictor) on the Set 2 sample (i.e. just those patients who did have their ethnicity recorded).

\section{Results}

\section{Descriptive statistics}

Table B1 and Figures B1 and B2 in Appendix B present the descriptive statistics of the LoS in ICU broken down by the predictors used in the model. In these figures we also break down the results by either of the outcomes (death or discharge/transfer) and censoring.

Among the patients with censored LoS, long ICU stays were more common, with a mean of around twice that of those who died or were discharged. The later the admission to ICU date, the more censored cases we observed (Table B1: $n=28$ before 25 March; $n=265$ afterwards).

For patients younger than seventy-five years old with known outcomes, we observed a mean ICU LoS of approximately thirteen days, whereas for those seventy-five or over this was around nine days. We observed a decreasing mean LoS for patients with known outcomes with the three periods of ICU admission. Non-White patients had slightly longer LoS in ICU; whereas those needing ECMO stayed around four days longer in the ICU. There was also a considerable variation in the observed LoS for all subgroups; overall the standard deviation (SD) was ten days for those with known outcomes and sixteen for those without.

\section{AFT models}

We found that the assumption of a log-normal baseline hazard function, which allows for a non-monotonic, peaked hazard, gave the best (lowest) AIC (7,937.42, compared to $8,021.42$

\footnotetext{
${ }^{5}$ Information about updates to the guidelines can be found at https://www.nice.org.uk/guidance/ng159/chapter/Update-information
} 
for the equivalent Weibull model; see Tables C1 and C2). The interaction effects (between admission period with age and health-risk score, and between age and sex) did not improve the AIC (7,949.64 and 7,953.82 for the log-normal models with interactions; see Tables $\mathrm{C} 3$ and $\mathrm{C} 4$, respectively), so we report the results from the log-normal model with maineffects only in Table 2. Results for the other tested models, including Weibull and models with interactions, are shown in Appendix C.

Table 2 shows the model coefficients, which represent the effects on mean log LoS associated with the predictors. Patients admitted during the early admission period had a significantly higher average LoS compared to those admitted after 7 April (at a significance level $\alpha=5 \%$ ). There was weak evidence $(p$ value $=0.055)$ that admissions during the middle period (25 March to 7 April) had longer LoS. The 50-65 age group had the longest LoS, with all other age groups having shorter estimated LoS in comparison. Treatment with ECMO was strongly associated with longer $\operatorname{LoS}\left(e^{0.40}=1.49\right.$, i.e. around $49 \%$ longer than non-ECMO patients, after controlling for the other predictors). We also found a negative association between the co-morbid risk factors score and ICU LoS. Patients with the lowest score (i.e. least number of co-morbid risk factors, weighted for their co-occurrence in the Rasch model) had LoS around 6\% longer than average; those with the highest score had LoS shorter by around $14 \%$. There was only very weak evidence of an association between sex and the LoS.

We used the results from the model shown in Table 2 to predict LoS for each patient (Table 3). The overall mean predicted LoS for the sample was 16.5 days (SD 3.0). The variation in model predicted LoS associated with the two main significant predictors, admission period and age, are shown in Figures $1 \mathrm{a}$ and $1 \mathrm{~b}$.

Figure 1a shows that the age group with the shortest predicted mean LoS, 10.7 days (SD 1.0), was the oldest group (75 and older). Inspection of the final outcomes for this group showed that it was the one with the highest proportion of deaths (over $70 \%$ of the 304 non-censored cases). The next-shortest average LoS, of 15.4 days (SD 2.3) was for the youngest group (less than 50 years old). This younger group had the lowest proportion of deaths $(18 \%$ of noncensored cases). The group with the longest predicted LoS was the 50-64 year old group, with a mean of 18.4 days (SD 1.9).

Figure $1 \mathrm{~b}$ shows that the later the admission period, the shorter the LoS on average, with the early period having a mean predicted LoS of 18.4 days (SD 3.4) and the latest admission period, after April 7th, having a mean predicted LoS of 15.4 days (SD 2.6). The LoS distributions by admission period were notably bi-modal, with a small peak with relatively short LoS for each period. This was associated with the patients in the oldest age group, which, as shown in Figure 1a, had the shortest average predicted LoS by some margin.

The inclusion of ethnicity (based on a smaller sample size $n=2,514)$ did not improve the model fit $(\mathrm{AIC}=6,572.26$ without ethnicity vs $\mathrm{AIC}=6,574.48$ with ethnicity). Moreover, the pattern of findings from the model without ethnicity as a predictor was virtually the same as in the model without ethnicity but with the full sample size $n=3,049$ (see Table C5 and Figure $\mathrm{C} 1$ ).

\section{Discussion}

Our estimated mean LoS for ICU patients was over sixteen days. The median, at nearly eleven days, was similar to the values reported by ICNARC [7] for England (twelve days for survivors, nine for non-survivors; or 10.8 for either of the outcomes on 3 July; 10.1 on 5 June). Our estimates adjust for the censored cases, who have longer than average LoS in the sample (Table B1).

Comparisons with other countries are less informative, because of differences in the populations and health-care systems, but our estimated LoS were longer than [5] for Italy, (2020; median nine days), and [12] for China (median of eight days based on a compilation of forty-six different studies);

Table 2: Log-normal Accelerated Failure Time model with main effects results

\begin{tabular}{lcrr}
\hline Variable & Coefficient & Robust SE & \multicolumn{1}{c}{$\boldsymbol{p}$} \\
\hline female & -0.05 & 0.04 & 0.193 \\
admission period & (reference: after 07/04) & 0.002 \\
before 25/03 & 0.21 & 0.07 & 0.055 \\
$25 / 03-07 / 04$ & 0.09 & 0.05 & $<0.001$ \\
age group & (reference: 50-64) & 0.021 \\
$<50$ & -0.22 & 0.06 & $<0.001$ \\
$65-74$ & -0.12 & 0.05 & 0.047 \\
$75+$ & -0.55 & 0.06 & 0.004 \\
risk score & -0.06 & 0.03 & $<0.14$ \\
ecmo & 0.40 & 0.01 \\
intercept & 2.42 & 0.05 & $<0.001$ \\
log $(\sigma)$ & -0.07 & 0.02 & \\
$n$ & 3,049 & & \\
AIC & $7,937.42$ & & \\
\hline
\end{tabular}

Source: own elaboration using CHESS data. 
Table 3: Predicted length-of-stay in days based on the log-normal model with main effects

\begin{tabular}{|c|c|c|c|c|c|}
\hline \multirow{2}{*}{ Age } & \multirow{2}{*}{ Measure } & \multicolumn{3}{|c|}{ Admission period } & \multirow{2}{*}{ Total } \\
\hline & & $<25 / 03$ & $25 / 03-07 / 04$ & $>07 / 04$ & \\
\hline \multirow[t]{4}{*}{$18-50$} & mean & 17.3 & 15.5 & 14.8 & 15.4 \\
\hline & median & 11.2 & 10.1 & 9.6 & 10.0 \\
\hline & SD & 1.9 & 1.8 & 2.5 & 2.3 \\
\hline & $\mathrm{N}$ & 79 & 306 & 297 & 682 \\
\hline \multirow[t]{4}{*}{$50-64$} & mean & 21.3 & 18.8 & 17.2 & 18.4 \\
\hline & median & 13.8 & 12.2 & 11.2 & 12.0 \\
\hline & SD & 2.0 & 1.4 & 1.2 & 1.9 \\
\hline & $\mathrm{N}$ & 141 & 646 & 535 & 1,322 \\
\hline \multirow[t]{4}{*}{$65-74$} & mean & 18.9 & 16.7 & 15.1 & 16.5 \\
\hline & median & 12.3 & 10.9 & 9.8 & 10.7 \\
\hline & SD & 1.3 & 0.8 & 0.8 & 1.5 \\
\hline & $\mathrm{N}$ & 106 & 360 & 260 & 726 \\
\hline \multirow[t]{4}{*}{$75+$} & mean & 12.3 & 10.9 & 9.7 & 10.7 \\
\hline & median & 8.0 & 7.1 & 6.3 & 7.0 \\
\hline & SD & 0.5 & 0.5 & 0.4 & 1.0 \\
\hline & $\mathrm{N}$ & 61 & 140 & 118 & 319 \\
\hline \multirow[t]{4}{*}{ Total } & mean & 18.4 & 16.8 & 15.4 & 16.5 \\
\hline & median & 12.0 & 10.9 & 10.0 & 10.7 \\
\hline & SD & 3.4 & 2.7 & 2.6 & 3.0 \\
\hline & $\mathrm{N}$ & 387 & 1,452 & 1,210 & 3,049 \\
\hline
\end{tabular}

Note: "mean" denotes a mean of the predicted mean LoS for all individual patients; "median" denotes a mean of the predicted median LoS for all patients. Source: own elaboration using CHESS data.

some studies reported median LoS in ICU as short as five days $[13,14]$.

We found that ICU LoS was decreasing over our study period, with patients admitted before 25 March having LoS five days longer on average than those admitted after 7 April. There was no evidence of any differentiation of this effect between patients in various age groups, or with pre-existing risk factors.

Unfortunately, the reasons for this change cannot be determined from the CHESS data, as several different and likely influential processes were confounded (except patient age, which we adjusted for): changes in the characteristics of patients contracting Covid-19 because of the stage of the epidemic, changes in who was admitted to ICU because of guideline changes and shielding introduced by the UK Government [33] around 21 March 2020 (i.e., during the first wave of the pandemic), and changes in the treatment and management of Covid-19 patients [17]. The first of the above possible explanations is further obscured by the non-uniform spread of the Covid-19 across the regions of England, with London and the Midlands being affected first. The changes to the guidelines are likely to have a modest effect as the final decisions on whether to elevate care to intensive are ultimately taken by clinicians. Also, general compliance with advice on shielding of vulnerable groups might have influenced the composition of patients admitted to specific hospitals and ICUs [34]. Further, at no point was the capacity of the ICUs in England overwhelmed by the number of patients, according to the NHS data on bed availability and occupancy [35]. However, some of the hospitals (e.g. in London) were close to the limit.
It is not clear from the CHESS data whether this trend in shortening ICU LoS for Covid-19 patients was approaching an asymptote, but the balance among the influences on LoS may shift as the pandemic moves on from the first peak, possibly lengthening the LoS again in future waves of the pandemic.

We found that LoS was not monotonic with age (i.e. either increasing or decreasing), unlike the relationship between age and Covid-19 severity and mortality [7]. While the group with the shortest LoS was the oldest one, the group with the longest LoS was the "younger" middle-aged group between 50 and 64 years. The middle-aged groups in our sample had roughly equal proportions of those who died and those who were discharged from ICU (for the patients with non-censored outcomes), i.e. the final outcome for these patients was likely the most uncertain on admission to ICU. Our sample was highly selective (i.e. patients ill enough to be in ICU with Covid-19) and this has the potential to bias our results, particularly with regard to age. For example, for patients with the same level of severe symptoms of Covid-19, younger patients were more likely to be hospitalized than older, frailer patients [36].

We found no evidence in the CHESS data that LoS in ICU is related to sex or ethnicity of the patient, and this was consistent across all tested models. Also, the shorter LoS found in more recent periods was not selectively affecting e.g. non-White patients only nor patients with certain pre-existing conditions score.

We argue that the median LoS better describes the central tendency of LoS, but mean LoS might be better to be used in planning of ICU capacity. In our case, mean LoS was five days longer than the median (Table 3 ); this reveals a tail of the distribution of mean predicted LoS with very long LoS, as 
Figure 1: Distribution of mean predicted LoS in ICU

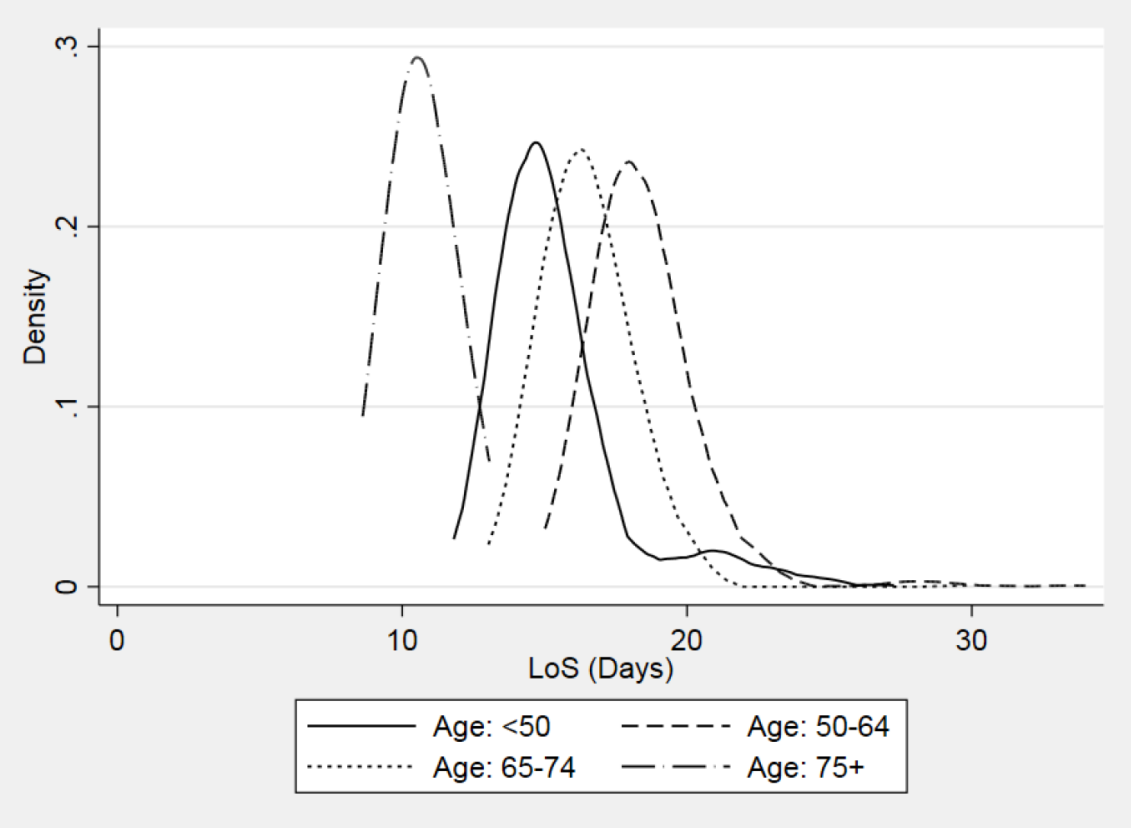

(a) Model-predicted LoS, by age group

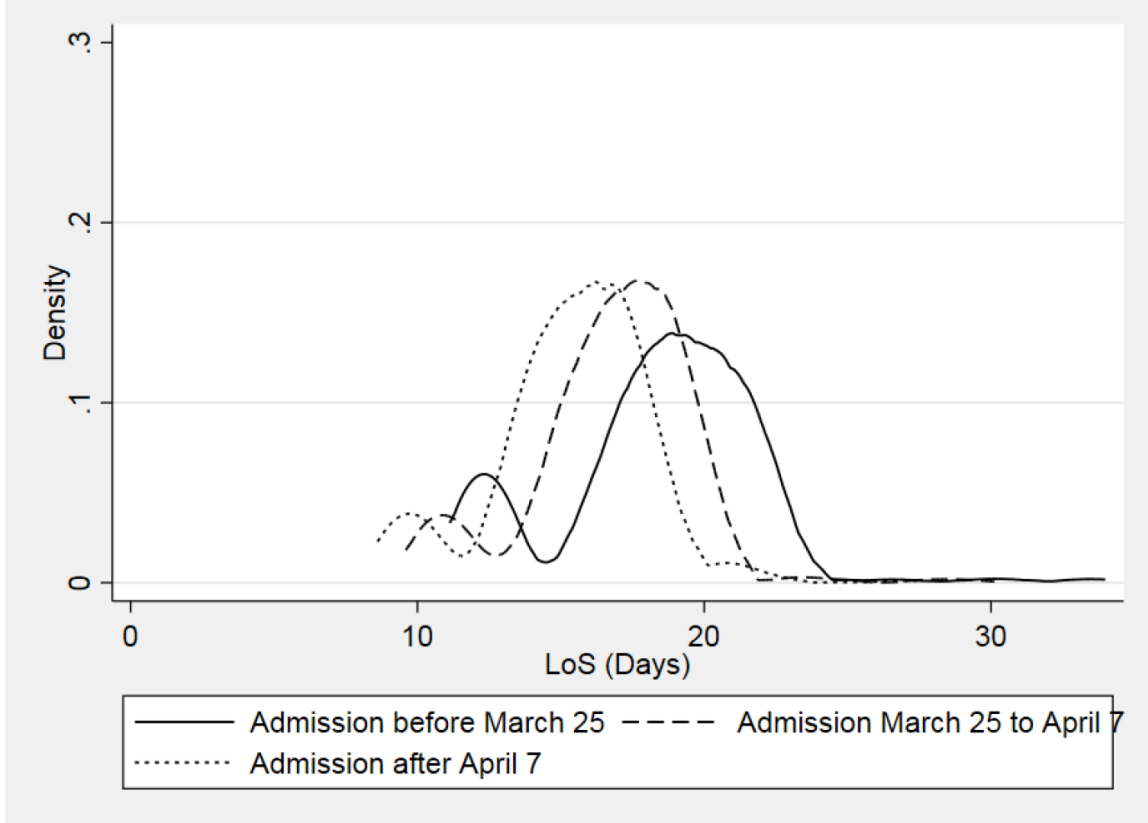

(b) Model-predicted LoS, by admission period.

Source: own elaboration using CHESS data and log-normal AFT model.

also shown in Figure 1. This ought to be taken into account in situations when the infections are increasing exponentially during early phases of the pandemic or potential further waves.

\section{Limitations}

The individual-level CHESS data, while in principle being a census of all patients with Covid-19 in England, had severe missingness issues. For example, when compared with the NHS Situation Reports (SitRep) deaths, they captured only around $13 \%$ of deaths in hospitals. Those reported cases suffered from missingness of predictors and other key information, especially dates of admission to ICU and final outcome, as well as ethnicity. The missingness patterns varied by NHS trust and geography; London and the Midlands had the highest percentages of missingness, which might have been due to these regions experiencing the peak of the pandemic earlier than elsewhere. Further, each NHS trust operates their IT system autonomously, which may lead to discrepancies in coverage and quality of collecting non-routine data, such as individual-level CHESS data. These data-collection systems might have been under various levels of pressure during the peak of the pandemic.

Although our statistical models were suitable for adjusting the observed overall LoS for censoring, they did not provide LoS estimates separately for those in ICU who eventually died and those who were discharged. The models only capture the "net" effect of predictors on LoS, and these effects may 
be countervailing for different outcomes, e.g. old age may be associated with a shorter LoS for eventual death but a longer LoS for eventual recovery. To overcome this limitation, more sophisticated models can be used. These methods include AFT models for "competing" outcomes [37] and multistate models [38], that allow for finer-grained analysis of the influences on ICU LoS and the different effects of predictors on death vs. discharge. However, these approaches would require data with nearly perfect coverage of all events, which was not the case with the CHESS data.

\section{Conclusion}

Covid-19 Hospitalisation in England Surveillance System (CHESS) data are limited due to case and variable missingness. Future planning should enable more automated data collection for clinical staff, e.g. based on existing patient data, with a common standard of a minimum set of patient characteristics that would permit national comparisons. Also, more detailed information about inclusion and exclusion criteria for the NHS trusts participating in the data collection [18] would help alleviate potential biases resulting from the selective samples.

Our analyses of the CHESS data suggest that using the mean LoS, as compared with median, might be better suited as a measure for assessing and planning ICU bed capacity, because the median neglects the severe skew of the distribution of LoS. This is important when considering subsequent waves of Covid-19 cases and potential pressure on ICU resources. We found that the most informative pre-admission predictors of LoS were not necessarily the same ones that predict severity of Covid-19 outcomes, with the exception of age. The weak effect of comorbidities and the likely potential for them to be influenced by clinical practice suggests that using them to make predictions may also require using more recent data and improved models. When considering the utility of such predictors in planning ICU bed capacity, we note that changes in ICU admission policy are likely to be confounded with improvements in clinical knowledge of Covid-19, rendering their utility labile over time.

\section{Acknowledgements}

The authors gratefully acknowledge support from Policy@ Manchester within The University of Manchester, as part of the QR SPF grant monies allocation from UKRI Research England. The corresponding author also gratefully acknowledges the funding received from the Engineering and Physical Sciences Research Council, grant Ref. EP/ V027468/1.

\section{Statement on conflicts of interest}

All authors declare that they have no conflicts of interest.

\section{Ethics statement}

Public Health England gathers data from National Health Service microbiology laboratories, storing it in the SGSS database for epidemiological analysis, an activity permitted under Section 251 of the National Health Service Act 2006, which allows processing of patient data for defined purposes, including public health surveillance such as CHESS data. This work was prepared for the Scientific Pandemic Influenza Group on Modelling (SPI-M) that advises the UK's Department for Health and Social Care on its COVID-19 response.

\section{References}

1. Chinazzi M, Davis JT, Ajelli M, Gioannini C, Litvinova M, Merler S, et al. The effect of travel restrictions on the spread of the 2019 novel coronavirus (COVID-19) outbreak. Science 2020;368(6489):395-400. https://doi.org/10.1126/science.aba9757

2. Flaxman S, Mishra S, Gandy A, Unwin HJT, Mellan TA, Coupland $H$, et al. Estimating the effects of nonpharmaceutical interventions on COVID-19 in Europe. Nature 2020;584(7820):257-61. URL: https://www. nature.com/articles/s41586-020-2405-7. https://doi. org/10.1038/s41586-020-2405-7

3. Dorigatti I, Okell L, Cori A, Imai N, Baguelin M, Bhatia S, et al. Report 4: severity of 2019-novel coronavirus (ncov). Tech. Rep.; Imperial College London, London; 2020. https://doi.org/10.25561/77154

4. Ferguson N, Laydon D, Nedjati Gilani G, Imai N, Ainslie K, Baguelin $M$, et al. Report 9: Impact of non-pharmaceutical interventions (npis) to reduce covid19 mortality and healthcare demand. Tech. Rep.; Imperial College London; 2020. https://doi.org/10.25561/77482

5. Grasselli G, Zangrillo A, Zanella A, Antonelli M, Cabrini $L$, Castelli A, et al. Baseline characteristics and outcomes of 1591 patients infected with SARS-CoV-2 admitted to ICUs of the Lombardy Region, Italy. Journal of the American Medical Association 2020;323(16):1574-81. URL: https://jamanetwork.com/journals/jama/articleabstract/2764365. https://doi.org/10.1001/jama.2020. 5394

6. Zhou F, Yu T, Du R, Fan G, Liu Y, Liu Z, et al. Clinical course and risk factors for mortality of adult inpatients with COVID-19 in Wuhan, China: a retrospective cohort study. The Lancet 2020;395(10229):1054-62. URL: https://www.sciencedirect.com/science/article/pii/S014 0673620305663. https://doi.org/10.1016/S0140-6736 (20)30566-3

7. Intensive Care National Audit \& Research Centre. ICNARC report on COVID-19 in critical care, 03 July 2020. 2020. URL: https://www.icnarc.org/Data Services/Attachments/Download/da626009-65bd-ea119127-00505601089b 
8. Xie J, Tong Z, Guan X, Du B, Qiu H, Slutsky AS. Critical care crisis and some recommendations during the COVID-19 epidemic in China. Intensive Care Medicine 2020;46:837-40. https://doi.org/10.1007/ s00134-020-05979-7

9. Mizumoto K, Kagaya K, Zarebski A, Chowell G. Estimating the asymptomatic proportion of coronavirus disease 2019 (covid-19) cases on board the diamond princess cruise ship, yokohama, japan, 2020. Eurosurveillance 2020;25(10): 2000180. https://doi.org/10.2807/1560-7917.ES. 2020.25.10.2000180

10. Wu Y, Xu X, Chen Z, Duan J, Hashimoto K, Yang $L$, et al. Nervous system involvement after infection with COVID-19 and other coronaviruses. Brain, behavior, and immunity 2020;87:18-22. URL: https://www.sciencedirect.com/science/article/abs/pii/ S0889159120303573. https://doi.org/10.1016/j.bbi.2020. 03.031

11. Yang $X, Y u Y, X u$ J, Shu $H$, Liu $H$, Wu $Y$, et al. Clinical course and outcomes of critically ill patients with SARS-CoV-2 pneumonia in Wuhan, China: a single-centered, retrospective, observational study. The Lancet Respiratory Medicine 2020;8:475-81. https://doi.org/10.1016/S2213-2600(20)30079-5

12. Rees EM, Nightingale ES, Jafari Y, Waterlow NR, Clifford S, B. Pearson CA, et al. COVID-19 length of hospital stay: a systematic review and data synthesis. BMC Medicine 2020;18(1):270. URL: https://doi.org/10.1186/s12916020-01726-3

13. Thomas-Rüddel D, Winning J, Dickmann $P$, Ouart D, Kortgen A, Janssens $U$, et al. Coronavirus disease 2019 (COVID-19): update for anesthesiologists and intensivists March 2020. Der Anaesthesist 2020;:1-10. https://doi.org/10.1007/s00101-020-00760-3

14. Wang D, Hu B, Hu C, Zhu F, Liu X, Zhang J, et al. Clinical characteristics of 138 hospitalized patients with 2019 novel coronavirus-infected pneumonia in Wuhan, China. Journal of the American Medical Association 2020;323(11):1061-9. URL: https://jamanetwork.com/journals/jama/articleabstract/2761044. https://doi.org/10.1001/jama.2020. 1585

15. Guan $\mathrm{Wj}, \mathrm{Ni} \mathrm{Zy}, \mathrm{Hu} \mathrm{Y}$, Liang $\mathrm{Wh}, \mathrm{Ou} \mathrm{Cq}$, $\mathrm{He} J \mathrm{x}$, et al. Clinical characteristics of coronavirus disease 2019 in China. The New England Journal of Medicine 2020;382(18):1708-20. https://doi.org/ 10.1056/NEJMoa2002032

16. National Institute for Health and Care Excellence COVID-19 rapid guideline: critical care in adults. 2020. URL: https://www.nice.org.uk/guidance/ng159

17. Bamford P, Bentley A, Dean J, Whitmore D, Wilson-Baig $\mathrm{N}$. ICS guidance for prone positioning of the conscious COVID patient 2020. Intensive Care Society; 2020. URL: https://emcrit.org/wp-content/uploads/2020/04/202004-12-Guidance-for-conscious-proning.pdf

18. Public Health England. Weekly Coronavirus Disease 2019 (COVID- 19) Surveillance Report. 2020. URL: https://tinyurl.com/phe-cov19surv-wk23

19. National Health Service. Services: Acute Trust List. 2020. URL: https://www.nhs.uk/Services/; accessed $27 / 11 / 2020$

20. Molina JAD, Seow E, Heng BH, Chong WF, Ho $B$. Outcomes of direct and indirect medical intensive care unit admissions from the emergency department of an acute care hospital: a retrospective cohort study. British Medical Journal open 2014;4(11):e005553. https://doi.org/10.1136/bmjopen-2014-005553

21. Hashemi N, Viveiros K, Redd WD, Zhou JC, McCarty TR, Bazarbashi AN, et al. Impact of chronic liver disease on outcomes of hospitalized patients with COVID-19: A multicentre United States experience. Liver International 2020;40(10):2515-21. https://doi.org/10.1111/liv.14583

22. Jordan RE, Adab P, Cheng KK. Covid-19: risk factors for severe disease and death. British Medical Journal 2020;368:m1198. URL: https:// www.bmj.com/content/368/bmj.m1198. https://doi. org/10.1136/bmj.m1198. arXiv:https://www.bmj. com/content/368/bmj.m1198.full.pdf

23. Phua J, Weng L, Ling L, Egi M, Lim CM, Divatia JV, et al. Intensive care management of coronavirus disease 2019 (COVID-19): challenges and recommendations. The Lancet Respiratory Medicine 2020;8:506-17. URL: https://www.sciencedirect.com/science/article/abs/pii/ S2213260020301612. https://doi.org/10.1016/S22132600(20)30161-2

24. Porcheddu R, Serra C, Kelvin D, Kelvin N, Rubino S. Similarity in case fatality rates (CFR) of COVID19/SARS-COV-2 in Italy and China. The Journal of Infection in Developing Countries 2020;14(02): 125-8. URL: https://jidc.org/index.php/journal/article/ view/32146445. https://doi.org/10.3855/jidc.12600

25. Wu Z, McGoogan JM. Characteristics of and important lessons from the coronavirus disease 2019 (COVID-19) outbreak in China: summary of a report of 72314 cases from the Chinese Center for Disease Control and Prevention. Journal of the American Medical Association 2020;323(13):1239-42. URL: https://jama network.com/journals/jama/article-abstract/2762130. https://doi.org/10.1001/jama.2020.2648

26. Wolfe EW, Smith JE. Instrument development tools and activities for measure validation using Rasch models: part II-validation activities. Journal of Applied Measurement 2007;8(2):204-34.

27. Ohannessian R, Gustin MP, Bénet T, Gerbier-Colomban S, Girard R, Argaud L, et al. Estimation of extra length of stay attributable to hospital-acquired infections 
in adult ICUs using a time-dependent multistate model. Critical Care Medicine 2018;46(7):1093-8. https://doi.org/10.1097/CCM.0000000000003131

28. Bréchot N, Luyt CE, Schmidt M, Leprince P, Trouillet $\mathrm{JL}$, Léger $\mathrm{P}$, et al. Venoarterial extracorporeal membrane oxygenation support for refractory cardiovascular dysfunction during severe bacterial septic shock. Critical Care Medicine 2013;41(7):1616-26. https://doi.org/10.1097/CCM.0b013e31828a2370

29. Peek GJ, Mugford M, Tiruvoipati R, Wilson A, Allen $E$, Thalanany $M M$, et al. Efficacy and economic assessment of conventional ventilatory support versus extracorporeal membrane oxygenation for severe adult respiratory failure (CESAR): a multicentre randomised controlled trial. The Lancet 2009;374(9698):1351-63. https://doi.org/10.1016/S0140-6736(09)61069-2

30. Alhazzani W, Møller MH, Arabi YM, Loeb M, Gong MN, Fan E, et al. Surviving Sepsis Campaign: guidelines on the management of critically ill adults with Coronavirus Disease 2019 (COVID-19). Intensive Care Medicine 2020;46:854-87. https://doi. org/10.1007/s00134-020-06022-5

31. StataCorp. Stata Statistical Software: Release 16. College Station, TX: StataCorp LLC. 2019.

32. Burnham KP, Anderson DR. Multimodel inference: understanding AIC and BIC in model selection. Sociological methods \& research 2004;33(2):261-304. https://doi.org/10.1177/0049124104268644

33. UK Government. Guidance on shielding and protecting people who are clinically extremely vulnerable from COVID-19. 2020. URL: https:// www.gov.uk/government/publications/guidance-onshielding-and-protecting-extremely-vulnerable-personsfrom-covid- guidance-on-shielding-and-protectingextremely-vulnerable-persons-from-covid- accessed 31/07/2020.
34. Office for National Statistics. Coronavirus and shielding of clinically extremely vulnerable people in England: 28 may to 3 June 2020. 2020. URL: https://www.ons.gov.uk/peoplepopulationandcommunity/ healthandsocialcare/conditionsanddiseases/ bulletins/coronavirusandshieldingo fclinicallyextremelyvulnerablepeople inengland/28mayto3june2020; accessed 15/07/2020.

35. National Health Service. Bed Availability and Occupancy. 2020. URL: https://www.england.nhs. uk/statistics/statistical-work-areas/bed-availabilityand-occupancy/

36. Griffith G, Morris TT, Tudball M, Herbert A, Mancano G, Pike L, et al. Collider bias undermines our understanding of COVID-19 disease risk and severity. Nature Communications 2020;5749. https:// doi.org/10.1038/s41467-020-19478-2

37. Lee S, Lewbel A. Nonparametric identification of accelerated failure time competing risks models. Econometric Theory 2013;29(5):905-19. URL: https://www.jstor.org/stable/24534479. https://doi. org/10.1017/S0266466612000795

\section{Abbreviations}

AIC: $\quad$ Akaike Information Criterion

CHESS: Covid-19 Hospitalisation in England Surveillance System

ECMO: Extracorporeal Membrane Oxygenation

HAI: Hospital-Acquired Infection

ICU: Intensive Care Unit LoS Length of Stay

NHS: National Health Service SD Standard deviation 